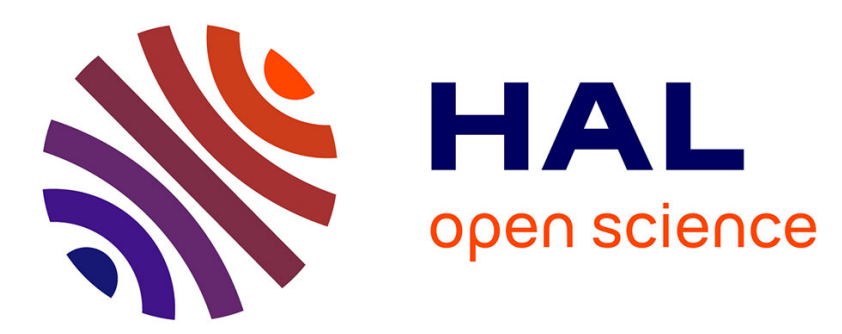

\title{
Delayed stabilization of parabolic PDEs via augmented Lyapunov functionals and Legendre polynomials
} Jin Zhang, Wen Kang, Emilia Fridman, Alexandre Seuret

\section{To cite this version:}

Jin Zhang, Wen Kang, Emilia Fridman, Alexandre Seuret. Delayed stabilization of parabolic PDEs via augmented Lyapunov functionals and Legendre polynomials. Systems and Control Letters, 2021, 156, pp.105003. 10.1016/j.sysconle.2021.105003 . hal-03395170

\author{
HAL Id: hal-03395170 \\ https://hal.laas.fr/hal-03395170
}

Submitted on 22 Oct 2021

HAL is a multi-disciplinary open access archive for the deposit and dissemination of scientific research documents, whether they are published or not. The documents may come from teaching and research institutions in France or abroad, or from public or private research centers.
L'archive ouverte pluridisciplinaire HAL, est destinée au dépôt et à la diffusion de documents scientifiques de niveau recherche, publiés ou non, émanant des établissements d'enseignement et de recherche français ou étrangers, des laboratoires publics ou privés. 


\title{
Delayed stabilization of parabolic PDEs via augmented Lyapunov functionals and Legendre polynomials *
}

\author{
Jin Zhang ${ }^{\text {a }}$, Wen Kang ${ }^{\mathrm{b}}$, Emilia Fridman ${ }^{\mathrm{a}}$, Alexandre Seuret ${ }^{\mathrm{c}}$ \\ ${ }^{\text {a }}$ School of Electrical Engineering, Tel Aviv University, Tel Aviv 69978, Israel \\ ${ }^{\mathrm{b}}$ School of Automation and Electrical Engineering, University of Science and Technology Beijing, Beijing 100083, China \\ ${ }^{\mathrm{c}} L A A S-C N R S$, Université de Toulouse, CNRS, France
}

\begin{abstract}
We first study stabilization of heat equation with globally Lipschitz nonlinearity. We consider the point measurements with constant delay and use spatial decomposition. Inspired by recent developments in the area of ordinary differential equations (ODEs) with time-delays, for the stability analysis, we suggest an augmented Lyapunov functional depending on the state derivative that is based on Legendre polynomials. Global exponential stability conditions are derived in terms of linear matrix inequalities (LMIs) that depend on the degree $\mathcal{N}$ of Legendre polynomials. The stability conditions form a hierarchy of LMIs: if the LMIs hold for $\mathcal{N}$, they hold for $\mathcal{N}+1$. The dual observer design problem with constant delay is also formulated. We further consider stabilization of Korteweg-de Vries-Burgers (KdVB) equation using the point measurements with constant delay. Due to the third-order partial derivative in KdVB equation, the Lyapunov functionals that depend on the state derivative are not applicable here, which is different from the case of heat equation. We suggest a novel augmented Lyapunov functional depending on the state only that leads to improved regional stability conditions in terms of LMIs. Finally, numerical examples illustrate the efficiency of the method.
\end{abstract}

Key words: Parabolic PDEs, Time-delays, Lyapunov functionals, Bessel-Legendre inequality, LMIs.

\section{Introduction}

Control of partial differential equations (PDEs), e.g. heat equation, becomes an active research topic $[4,17]$. It is of interest to design a control law for PDEs using delayed inputs/outputs. Constructive conditions in terms of LMIs for delayed control of PDEs were presented in $[7,8]$. The derived conditions allow to give an upper bound on the delay preserving the performance (e.g. exponential decay rate). Moreover, KdVB equation has been derived as the governing evolution equation for waves propagating in fluid-filled

\footnotetext{
^ This work was supported by Israel Science Foundation under Grant 673/19, C. and H. Manderman Chair on System Control at Tel Aviv University, the Planning and Budgeting Committee (PBC) Fellowship from the Council for Higher Education, Israel, Joint Research Project HetCPS, Ministry of Science \& Technology of Israel and CNRS, France, National Natural Science Foundation of China under Grant 61803026, and Fundamental Research Funds for the Central Universities under Grant FRF-TP-20-039A2Z.

Email addresses: zhangjin1116@126.com (Jin Zhang), kangwen@amss.ac.cn (Wen Kang), emilia@tauex.tau.ac.il (Emilia Fridman), aseuret@laas.fr (Alexandre Seuret).
}

elastic or viscoelastic tubes incorporating the effects of dispersion, dissipation and nonlinearity [5]. The objective of the present work is the derivation of less conservative LMI conditions for the stability analysis of heat and KdVB equations with time-delay. In application to chain of subobservers as used in [1], such conditions will allow to reduce the order of the chain.

For the stability analysis of ODEs with time-delay, Jensen's inequality $[10,11]$ and Wirtinger-based integral inequality [19] were usually employed. Several contributions to derive less conservative integral inequalities for time-delay systems were provided in $[15,27]$. Recently, a novel integral inequality so-called Bessel-Legendre (B-L) inequality that encompasses Jensen's inequality and Wirtinger-based integral inequality as particular cases was introduced in [20] by using Legendre polynomials. The latter presented a hierarchy of LMI conditions that are competitive with $[10,11,15,19,27]$ in terms of conservatism and of complexity. In [3], stability analysis of a coupled ODE-heat equation was presented via a new B-L inequality.

In the present work, we consider stabilization of heat and KdVB equations in the presence of constant output de- 
lay. Note that in the case of constant delay, input delay can be always moved to output by changing the time. We first study stabilization of heat equation under the point measurements with constant delay by using spatial decomposition approach (as introduced in [8]). For the stability analysis of the closed-loop system, we suggest an augmented Lyapunov functional depending on the state derivative and that is based on Legendre polynomials. Such functionals extend the Lyapunov constructions of $[1,8]$. Sufficient stability conditions are derived in terms of LMIs that are parameterized by the degree $\mathcal{N}$ of the polynomials. The same LMIs are also applicable to the case of stabilization under the spatially averaged measurements with constan$\mathrm{t}$ delay. As a by-product, for the stability analysis of heat equation with constant state delay, we present the LMIs that appear to guarantee the exponential stability of delayed ODEs and that in the numerical example recover the analytical upper bounds on delay with a finite degree of the polynomials. We also formulate the dual observer design problem.

We further consider stabilization of KdVB equation under the point measurements with constant delay as studied in [13]. Due to the third-order partial derivative in KdVB equation, the Lyapunov functionals that depend on the state derivative are not applicable here, which is different from the case of heat equation. We suggest a novel augmented Lyapunov functional encompassing the Lyapunov functional introduced in [13] as a particular case that leads to improved regional stability conditions.

As in [20,21], the derived stability conditions form a hierarchy of LMIs: if the LMIs hold for $\mathcal{N}$, they hold for $\mathcal{N}+1$. By solving the LMIs, improved upper bounds on delay that preserve the stability are found. Finally, numerical examples illustrate the efficiency of the method. Some preliminary results for the scalar heat equation were presented in $[28]$.

\subsection{Notation and Preliminaries}

Throughout the paper, $\mathbb{R}^{n}$ denotes the $n$-dimensional Euclidean space with the vector norm $|\cdot|, \mathbb{R}^{n \times m}$ is the space of all $n \times m$ real matrices. The notation $P>0$, for $P \in \mathbb{R}^{n \times n}$ means that $P$ is symmetric and positive definite. $0_{n \times m}\left(0_{n}\right)$ stands for the matrix in $\mathbb{R}^{n \times m}\left(\mathbb{R}^{n \times n}\right)$ whose entries are zero. For any square matrix $X, \operatorname{He}\{X\}=X+X^{T} . L^{2}(0, l)$ stands for the Hilbert space of square integrable vector (or scalar for $n=1)$ functions $z:(0, l) \rightarrow \mathbb{R}^{n}$ with the norm $\|z\|_{L^{2}(0, l)}=\sqrt{\int_{0}^{l} z^{T}(x) z(x) d x} . \mathcal{H}^{i}(0, l)(i=1,2)$ are the Sobolev space: $\mathcal{H}^{i}(0, l)=\left\{z: \frac{d^{j} z}{d x^{j}} \in L^{2}(0, l) \forall 0 \leq j \leq i\right\}$ with the norm $\|z\|_{\mathcal{H}^{i}(0, l)}=\sqrt{\sum_{j=0}^{i}\left\|\frac{d^{j} z}{d x^{j}}\right\|_{L^{2}(0, l)}^{2}}$.

The notation $\left(\begin{array}{c}k \\ i\end{array}\right)$ refers to the binomial coefficients given by $\frac{k !}{(k-i) ! i !}$. Let $\mathcal{L}_{k}(s)\left(k \in \mathbb{N}_{0}\right)$ be the shifted Legendre polynomials over interval $[-h, 0]$ :

$$
\mathcal{L}_{k}(s)=\sum_{i=0}^{k}(-1)^{i+k}\left(\begin{array}{c}
k \\
i
\end{array}\right)\left(\begin{array}{c}
k+i \\
i
\end{array}\right)\left(\frac{s+h}{h}\right)^{i} .
$$

These polynomials satisfy the following properties:

Property 1.1 (i) Orthogonality:

$$
\forall k, i \in \mathbb{N}_{0}, \quad \int_{-h}^{0} \mathcal{L}_{k}(s) \mathcal{L}_{i}(s) d s= \begin{cases}0, & k \neq i \\ \frac{h}{2 k+1}, & k=i\end{cases}
$$

(ii) Boundary conditions:

$$
\forall k \in \mathbb{N}_{0}, \quad \mathcal{L}_{k}(0)=1, \quad \mathcal{L}_{k}(-h)=(-1)^{k} .
$$

(iii) Differentiation:

$$
\dot{\mathcal{L}}_{k}(s)= \begin{cases}0, & k=0 \\ \sum_{i=0}^{k-1} \frac{2 i+1}{h}\left(1-(-1)^{k+i}\right) \mathcal{L}_{i}(s), & k \geq 1\end{cases}
$$

We will employ extended Bessel-Legendre inequality that is obtained by integration in $x \in[0, l]$ of the inequality of Lemma 3 of [20]:

Lemma 1.1 Consider a function $z \in L^{2}\left([-h, 0] ; L^{2}(0, l)\right)$, and scalars $h>0$ and $l>0$. Then for any $n \times n$ matrix $R>0$, the following inequality holds for all $\mathcal{N} \in \mathbb{N}_{0}$ :

$$
\begin{aligned}
& \int_{0}^{l} \int_{-h}^{0} z^{T}(x, t+s) R z(x, t+s) d s d x \\
& \geq \sum_{k=0}^{\mathcal{N}} \frac{2 k+1}{h} \int_{0}^{l}\left(\Theta^{k}(x, t)\right)^{T} R \Theta^{k}(x, t) d x,
\end{aligned}
$$

where $\Theta^{k}(x, t)\left(k \in \mathbb{N}_{0}\right)$ correspond to the projection of $z(x, t+s)$ over $\mathcal{L}_{k}(s)$ given by $(1.1)$ :

$$
\Theta^{k}(x, t)=\int_{-h}^{0} \mathcal{L}_{k}(s) z(x, t+s) d s, \quad k \in \mathbb{N}_{0} .
$$

\section{Stabilization of heat equation with an output delay}

\subsection{Problem formulation}

Consider the following semilinear diffusion equation:

$$
\begin{aligned}
z_{t}(x, t)= & \triangle_{D} z(x, t)-\beta z_{x}(x, t)+A z(x, t) \\
& +\phi(z(x, t), x, t)+B \sum_{j=1}^{N} b_{j}(x) u_{j}(t), \\
& t \geq 0, \quad x \in[0, l], \quad l>0
\end{aligned}
$$

under the Dirichlet boundary conditions

$$
z(0, t)=z(l, t)=0 .
$$

Here $z(x, t)=\left[z^{1}(x, t), \ldots, z^{n}(x, t)\right]^{T} \in \mathbb{R}^{n}$ is the state, $u_{j}(t) \in \mathbb{R}^{r}(j=1, \ldots, N)$ are the control inputs, $A \in \mathbb{R}^{n \times n}$ and $B \in \mathbb{R}^{n \times r}$ are constant matrices and $\beta \in \mathbb{R}^{n \times n}$ is the diagonal matrix of convection coefficients. The diffusion term is given by

$$
\triangle_{D} z(x, t)=\left[\frac{\partial}{\partial x}\left(d_{1}(x) z_{x}^{1}(x, t)\right), \ldots, \frac{\partial}{\partial x}\left(d_{n}(x) z_{x}^{n}(x, t)\right)\right]^{T}
$$


with $d_{i}(x) \in \mathcal{C}^{1}$ satisfying $0<d_{0}^{i} \leq d_{i}(x)(i=1, \ldots, n)$ for $x \in[0, l]$.

Following [2], we assume that for some positive definite $\Psi \in \mathbb{R}^{n \times n}$, function $\phi \in \mathcal{C}^{1}$ satisfies

$$
\phi^{T}(z, x, t) \phi(z, x, t) \leq z^{T} \Psi z
$$

for all $z \in \mathbb{R}^{n}, x \in[0, l], t \geq 0$. It is well known that the open-loop system $(2.1)$ (with $u_{j}(t) \equiv 0$ ) under the Dirichlet boundary conditions (2.2) may become unstable if $\|\Psi\|$ in (2.4) is large enough (see [4] for $\left.\phi(z, x, t)=\phi_{M} z\right)$.

As in $[7,8]$, the control inputs $u_{j}(t)$ enter (2.1) through the shape functions

$$
b_{j}(x)=\left\{\begin{array}{ll}
1, & x \in \Omega_{j}, \\
0, & \text { otherwise, }
\end{array} \quad j=1, \ldots, N,\right.
$$

where $\Omega_{j}=\left[x_{j-1}, x_{j}\right)(j=1, \ldots, N)$ divide the domain $[0, l]$ into $N$ sub-intervals. Here points $0=x_{0}<x_{1}<$ $\cdots<x_{N}=l$ satisfy $x_{j}-x_{j-1}=\Delta_{j} \leq \Delta$, where $\Delta>0$ is a known parameter. Such shape functions correspond to actuation covering all the domain $[0, l]$.

Assume that $N$ sensors are placed in the middle of each interval $\Omega_{j}$ given by

$$
\bar{x}_{j}=\frac{x_{j-1}+x_{j}}{2}, \quad j=1, \ldots, N .
$$

In addition, the measurement is affected by a time-delay which is assumed to be constant, denoted hereafter by $h>0$. For the control design, our method works also for unknown but constant $h$ that belongs to a prescribed interval $\left[h_{1}, h_{2}\right]$. Then, point measurements are provided by $N$ sensors distributed over the whole domain $[0, l]$ :

$$
y_{j}(t)=\left\{\begin{array}{ll}
0, & t \leq h, \\
z\left(\bar{x}_{j}, t-h\right), & t>h,
\end{array} \quad j=1, \ldots, N .\right.
$$

Note that our model (2.1) may present a metal bar of the length of $l$ that is heated along its length. In this case we measure the temperature in the spatial points along the bar (see e.g. Example 1.1.2 in [4]). Another application of spatially sampled measurements was recently given in application to multi-agents in [25], where the measurements are provided by leaders placed in the points $\bar{x}_{j}$.

We design for system (2.1) a stabilizing controller

$$
u_{j}(t)=-K y_{j}(t), \quad t \geq 0, \quad j=1, \ldots, N
$$

with the gain $K \in \mathbb{R}^{r \times n}$. Denote by the errors between the delayed state $z(x, t-h)$ and point measurements $z\left(\bar{x}_{j}, t-\right.$ $h)$ :

$$
\begin{aligned}
f_{j}(x, t-h) & \triangleq z(x, t-h)-z\left(\bar{x}_{j}, t-h\right) \\
& =\int_{\bar{x}_{j}}^{x} z_{\xi}(\xi, t-h) d \xi, \quad x \in \Omega_{j}, \quad j=1, \ldots, N .
\end{aligned}
$$

Then the closed-loop system (2.1), (2.7), (2.8) has the form:

$$
\begin{aligned}
z_{t}(x, t)= & \triangle_{D} z(x, t)-\beta z_{x}(x, t)+A z(x, t) \\
& +\phi(z(x, t), x, t)-B K\left(1-\chi_{[0, h]}(t)\right) \\
& \times\left[z(x, t-h)-f_{j}(x, t-h)\right], \quad t \geq 0,
\end{aligned}
$$

where $x \in \Omega_{j}, j=1, \ldots, N$ and $\chi_{[0, h]}(t)$ denotes the characteristic function of the time interval $[0, h]$.

Now we consider the well-posedness of system (2.10). Let $\mathcal{H}_{0}^{1}(0, l)=\left\{\theta \in \mathcal{H}^{1}(0, l): \theta(0)=\theta(l)=0\right\}$ and

$$
\mathcal{D}(\mathcal{A})=\left\{\theta \in \mathcal{H}^{2}(0, l): \theta(0)=\theta(l)=0\right\} .
$$

By using the step method (see e.g. [8]), it can be shown that for any initial condition $z(\cdot, 0) \in \mathcal{H}_{0}^{1}(0, l)$, there exists a unique strong solution of (2.10) such that

$$
z \in L^{2}(0, \infty ; \mathcal{D}(\mathcal{A})) \cap \mathcal{C}\left([0, \infty) ; \mathcal{H}_{0}^{1}(0, l)\right)
$$

$z_{t} \in L^{2}\left(0, \infty ; L^{2}(0, \infty)\right)$ and equation (2.10) hold almost everywhere on $[h, \infty)$.

\subsection{Improved stability conditions}

For the stability analysis of system (2.10), we suggest the following augmented Lyapunov functional (that extends the Lyapunov constructions of $[1,8])$ :

$$
V_{\mathcal{N}}(t)=V_{\mathcal{P}_{\mathcal{N}}}+V_{P_{2}}+V_{S_{1}}+V_{S_{2}}+V_{R}, \quad t \geq h,
$$

where $\mathcal{N} \in \mathbb{N}_{0}$, and

$$
\begin{aligned}
& V_{\mathcal{P}_{\mathcal{N}}}=\int_{0}^{l} \zeta_{\mathcal{N}}^{T}(x, t) \mathcal{P}_{\mathcal{N}} \zeta_{\mathcal{N}}(x, t) d x \\
& V_{P_{2}}=\sum_{i=1}^{n} \int_{0}^{l} P_{2}^{i} d_{i}(x)\left(z_{x}^{i}(x, t)\right)^{2} d x \\
& V_{S_{1}}=\int_{0}^{l} \int_{t-h}^{t} e^{-2 \alpha(t-s)} z^{T}(x, s) S_{1} z(x, s) d s d x \\
& V_{S_{2}}=\int_{0}^{l} \int_{t-h}^{t} e^{-2 \alpha(t-s)} z_{x}^{T}(x, s) S_{2} z_{x}(x, s) d s d x, \\
& V_{R}=h \int_{0}^{l} \int_{t-h}^{t} e^{-2 \alpha(t-s)}(s-t+h) z_{s}^{T}(x, s) R z_{s}(x, s) d s d x
\end{aligned}
$$

with

$$
\zeta_{\mathcal{N}}(x, t)=\operatorname{col}\left\{z(x, t), \Theta^{0}(x, t), \ldots, \Theta^{\mathcal{N}-1}(x, t)\right\}
$$

and with $(\mathcal{N}+1) n \times(\mathcal{N}+1) n$ matrix $\mathcal{P}_{\mathcal{N}}, n \times n$ matrices $P_{2}=\operatorname{diag}\left\{P_{2}^{1}, \ldots, P_{2}^{n}\right\}>0, S_{1}>0, S_{2}>0, R>0$, a scalar $\alpha>0$ and $\Theta^{k}(x, t)(k=0, \ldots, \mathcal{N}-1)$ given by (1.6). The terms $V_{S_{1}}$ and $V_{R}$ are the PDE extensions of the standard Lyapunov functionals for delay-dependent analysis $[6,9]$. The term $V_{S_{2}}$ is introduced to compensate $\int_{0}^{l} z_{x}(x, t-h) d x$ (instead of Halanay's inequality employed in [8] for the case of time-varying delays). Note that $z(\cdot, s)$ for $s \in[0, h]$ in $(2.12)$ is defined as solution of $(2.10),(2.2)$ with the initial condition $z(\cdot, t) \in \mathcal{H}_{0}^{1}(0, l)$.

Theorem 2.1 For given positive scalars $h, l, \Delta, \alpha$ and $a$ positive integer $\mathcal{N}$, assume that there exist $(\mathcal{N}+1) n \times(\mathcal{N}+$ 1) $n$ matrix $\mathcal{P}_{\mathcal{N}}, n \times n$ matrices $P_{1}=\operatorname{diag}\left\{P_{1}^{1}, \ldots, P_{1}^{n}\right\}$, 
$P_{2}=\operatorname{diag}\left\{P_{2}^{1}, \ldots, P_{2}^{n}\right\}>0, S_{1}>0, S_{2}>0, R>0$, $W>0$ and a scalar $\lambda_{\phi}>0$ that satisfy

$$
\begin{gathered}
\overline{\mathcal{P}}_{\mathcal{N}}>0, \\
\Upsilon_{\mathcal{N}}+\Lambda_{\mathcal{N}}+U_{\mathcal{N}} \leq 0, \\
W-e^{-2 \alpha h} S_{2} \leq 0,
\end{gathered}
$$

where

$$
\begin{aligned}
& \overline{\mathcal{P}}_{\mathcal{N}}=\mathcal{P}_{\mathcal{N}}+\frac{1}{h} e^{-2 \alpha h} S_{1} \operatorname{diag}\{0,1, \ldots, 2 \mathcal{N}-1\}, \\
& \Upsilon_{\mathcal{N}}=\operatorname{He}\left\{G_{\mathcal{N}}^{T} \mathcal{P}_{\mathcal{N}} H_{\mathcal{N}}\right\}+2 \alpha G_{\mathcal{N}}^{T} \mathcal{P}_{\mathcal{N}} G_{\mathcal{N}} \\
& -e^{-2 \alpha h} \sum_{k=0}^{\mathcal{N}}(2 k+1) \Gamma_{\mathcal{N}}^{T}(k) R \Gamma_{\mathcal{N}}(k), \\
& G_{\mathcal{N}}=\left[\begin{array}{ccc}
I_{n} & 0_{n \times 5 n} & 0_{n \times \mathcal{N} n} \\
0_{\mathcal{N} n \times n} & 0_{\mathcal{N} n \times 5 n} & h I_{\mathcal{N} n}
\end{array}\right], \\
& H_{\mathcal{N}}=\left[\begin{array}{llll}
F_{\mathcal{N}}^{T} & \Gamma_{\mathcal{N}}^{T}(0) \ldots \Gamma_{\mathcal{N}}^{T}(\mathcal{N}-1)
\end{array}\right]^{T}, \\
& F_{\mathcal{N}}=\left[\begin{array}{lll}
0_{n} & I_{n} & 0_{n \times(\mathcal{N}+4) n}
\end{array}\right], \\
& \Gamma_{\mathcal{N}}(k)=\left[I_{n}, 0_{n \times 2 n},(-1)^{k+1} I_{n}, 0_{n \times 2 n}, \gamma_{\mathcal{N} k}^{0} I_{n}, \ldots, \gamma_{\mathcal{N} k}^{\mathcal{N}-1} I_{n}\right] \text {, } \\
& \gamma_{\mathcal{N} k}^{j}= \begin{cases}-(2 j+1)\left(1-(-1)^{k+j}\right), & \text { if } j \leq k, \\
0, & \text { if } j>k,\end{cases} \\
& U_{\mathcal{N}}=\frac{\pi^{2}}{l^{2}} \operatorname{diag}\left\{0_{3 n}, W-e^{-2 \alpha h} S_{2}, 0_{(\mathcal{N}+2) n}\right\}
\end{aligned}
$$

and $\Lambda_{\mathcal{N}}=\left\{\Lambda_{i j}\right\}$ is symmetric matrix composed of

$$
\begin{array}{lll}
\Lambda_{11}=\operatorname{He}\left\{P_{1} A\right\}+S_{1}+\lambda_{\phi} \Psi, & \Lambda_{12}=-P_{1}+A^{T} P_{2}, \\
\Lambda_{14}=-\Lambda_{16}=-P_{1} B K, & \Lambda_{15}=P_{1}, \\
\Lambda_{22}=-2 P_{2}+h^{2} R, & \Lambda_{23}=-P_{2} \beta, \\
\Lambda_{24}=-\Lambda_{26}=-P_{2} B K, & \Lambda_{25}=P_{2}, \\
\Lambda_{33}=S_{2}+\operatorname{He}\left\{D_{0}\left(\alpha P_{2}-P_{1}\right)\right\}, & \Lambda_{44}=-e^{-2 \alpha h} S_{1}, \\
\Lambda_{55}=-\lambda_{\phi} I_{n}, \quad \Lambda_{66}=-\frac{\pi^{2}}{\Delta^{2}} W, & \Lambda_{77}=0_{\mathcal{N} n}
\end{array}
$$

with $\Psi$ given by $(2.4)$ and $D_{0}=\operatorname{diag}\left\{d_{0}^{1}, \ldots, d_{0}^{n}\right\}$. Then there exists $M_{0}>0$ such that a unique strong solution of (2.10), (2.2) initialized with $z(\cdot, t) \in \mathcal{H}_{0}^{1}(0, l)$ for $t \in[0, h]$ satisfies the inequality

$$
\begin{aligned}
\|z(\cdot, t)\|_{\mathcal{H}_{0}^{1}(0, l)}^{2} \leq & M_{0} e^{-2 \alpha(t-h)} \int_{0}^{h}\left[\|z(\cdot, s)\|_{\mathcal{H}_{0}^{1}(0, l)}^{2}\right. \\
& \left.+\left\|z_{s}(\cdot, s)\right\|_{L^{2}(0, l)}^{2}\right] d s, \quad t \geq h,
\end{aligned}
$$

meaning that (2.10), (2.2) is exponentially stable with a decay rate $\alpha$. The bound in (2.19) is finite for the strong solutions of $(2.10),(2.2)$ on $[0, h]$. Moreover, if the strict inequalities (2.14)-(2.16) are feasible with $\alpha=0$, then (2.10) (2.2) is exponentially stable with a small enough decay rate $\alpha=\alpha_{0}>0$.
Proof: Consider Lyapunov functional $V_{\mathcal{N}}(t)$ given by (2.11). Lemma 1.1 gives a lower bound as follows

$$
\begin{aligned}
V_{\mathcal{N}}(t) \geq & \int_{0}^{l} \zeta_{\mathcal{N}}^{T}(x, t) \mathcal{P}_{\mathcal{N}} \zeta_{\mathcal{N}}(x, t) d x \\
& +\int_{0}^{l} \int_{t-h}^{t} e^{-2 \alpha(t-s)} z^{T}(x, s) S_{1} z(x, s) d s d x \\
\geq & \int_{0}^{l} \zeta_{\mathcal{N}}^{T}(x, t) \mathcal{P}_{\mathcal{N}} \zeta_{\mathcal{N}}(x, t) d x \\
& +e^{-2 \alpha h} \int_{0}^{l} \int_{t-h}^{t} z^{T}(x, s) S_{1} z(x, s) d s d x \\
\geq & \int_{0}^{l} \zeta_{\mathcal{N}}^{T}(x, t) \overline{\mathcal{P}}_{\mathcal{N}} \zeta_{\mathcal{N}}(x, t) d x
\end{aligned}
$$

Thus, the positivity of $V_{\mathcal{N}}(t)$ results from $\bar{P}_{\mathcal{N}}>0$ given by (2.14).

For $t \geq h$, differentiating $V_{P_{\mathcal{N}}}$ along (2.10) yields

$$
\dot{V}_{\mathcal{P}_{\mathcal{N}}}=2 \int_{0}^{l} \zeta_{\mathcal{N}}^{T}(x, t) \mathcal{P}_{\mathcal{N}} \dot{\zeta}_{\mathcal{N}}(x, t) d x
$$

where

$$
\dot{\zeta}_{\mathcal{N}}(x, t)=\operatorname{col}\left\{z_{t}(x, t), \Theta_{t}^{0}(x, t), \ldots, \Theta_{t}^{\mathcal{N}-1}(x, t)\right\} .
$$

Via (2.10) and (2.13), the definitions of $G_{\mathcal{N}}$ and $F_{\mathcal{N}}$ given by (2.17) yield

$$
\zeta_{\mathcal{N}}(x, t)=G_{\mathcal{N}} \eta_{\mathcal{N}, j}, \quad z_{t}(x, t)=F_{\mathcal{N}} \eta_{\mathcal{N}, j},
$$

where

$$
\begin{aligned}
& \quad \eta_{\mathcal{N}, j}=\operatorname{col}\left\{z(x, t), z_{t}(x, t), z_{x}(x, t), z(x, t-h),\right. \\
& \left.\quad \phi(z(x, t), x, t), f_{j}(x, t-h), \frac{1}{h} \Theta^{0}(x, t), \ldots, \frac{1}{h} \Theta^{\mathcal{N}-1}(x, t)\right\}
\end{aligned}
$$

with $f_{j}(x, t-h)$ defined by $(2.9)$.

Then, an integration by parts ensures that

$$
\begin{aligned}
\Theta_{t}^{k}(x, t)= & \left.\mathcal{L}_{k}(s) z(x, t+s)\right|_{s=-h} ^{0} \\
& -\int_{-h}^{0}\left[\frac{d}{d s} \mathcal{L}_{k}(s)\right] z(x, t+s) d s \\
= & \Gamma_{\mathcal{N}}(k) \eta_{\mathcal{N}, j}, \quad k=0, \ldots, \mathcal{N},
\end{aligned}
$$

where we applied (1.3) and (1.4) with $\Gamma_{\mathcal{N}}(k)$ given by (2.17). From $(2.20),(2.21)$ and $(2.22)$, it follows that

$$
\dot{V}_{\mathcal{P}_{\mathcal{N}}}=2 \sum_{j=1}^{N} \int_{x_{j-1}}^{x_{j}} \eta_{\mathcal{N}, j}^{T} G_{\mathcal{N}}^{T} \mathcal{P}_{\mathcal{N}} H_{\mathcal{N}} \eta_{\mathcal{N}, j} d x
$$

where $H_{\mathcal{N}}$ is given by $(2.17)$.

We have

$$
\begin{gathered}
\dot{V}_{P_{2}}=2 \sum_{i=1}^{n} \int_{0}^{l} P_{2}^{i} d_{i}(x) z_{x}^{i}(x, t) z_{x t}^{i}(x, t) d x, \\
\dot{V}_{S_{1}}+2 \alpha V_{S_{1}}=\int_{0}^{l} z^{T}(x, t) S_{1} z(x, t) d x \\
\quad-e^{-2 \alpha h} \int_{0}^{l} z^{T}(x, t-h) S_{1} z(x, t-h) d x, \\
\dot{V}_{S_{2}}+2 \alpha V_{S_{2}}=\int_{0}^{l} z_{x}^{T}(x, t) S_{2} z_{x}(x, t) d x \\
\quad-e^{-2 \alpha h} \int_{0}^{l} z_{x}^{T}(x, t-h) S_{2} z_{x}(x, t-h) d x .
\end{gathered}
$$

Note that $z_{x t}^{i}(i=1, \ldots, n)$ in $\dot{V}_{P_{2}}$ are well-defined as in Remark A.1 of [7]. 
Further by using Lemma 1.1 and taking into account (2.22), we obtain

$$
\begin{aligned}
& \dot{V}_{R}+2 \alpha V_{R}=h^{2} \int_{0}^{l} z_{t}^{T}(x, t) R z_{t}(x, t) d x \\
& \quad-h \int_{0}^{l} \int_{t-h}^{t} e^{-2 \alpha(t-s)} z_{s}^{T}(x, s) R z_{s}(x, s) d s d x \\
& \leq h^{2} \int_{0}^{l} z_{t}^{T}(x, t) R z_{t}(x, t) d x-e^{-2 \alpha h} \sum_{k=0}^{\mathcal{N}}(2 k+1) \\
& \quad \times \sum_{j=1}^{N} \int_{x_{j-1}}^{x_{j}} \eta_{\mathcal{N}, j}^{T} \Gamma_{\mathcal{N}}^{T}(k) R \Gamma_{\mathcal{N}}(k) \eta_{\mathcal{N}, j} d x .
\end{aligned}
$$

To cancel the term of the right hand side of (2.24), we employ the descriptor method $[6,8]$, where the right-hand side of

$$
\begin{aligned}
0= & 2 \int_{0}^{l}\left[z^{T}(x, t) P_{1}+z_{t}^{T}(x, t) P_{2}\right]\left[-z_{t}(x, t)\right. \\
& +\triangle_{D} z(x, t)-\beta z_{x}(x, t)+A z(x, t)+\phi(z(x, t), x, t) \\
& -B K z(x, t-h)] d x+2 \sum_{j=1}^{N} \int_{x_{j-1}}^{x_{j}}\left[z^{T}(x, t) P_{1}\right. \\
& \left.+z_{t}^{T}(x, t) P_{2}\right] B K f_{j}(x, t-h) d x
\end{aligned}
$$

with some $n \times n$ matrix $P_{1}=\operatorname{diag}\left\{P_{1}^{1}, \ldots, P_{1}^{n}\right\}$ is added to $\dot{V}_{\mathcal{N}}(t)+2 \alpha V_{\mathcal{N}}(t)$.

An integration by parts and substitution of the Dirichlet boundary conditions (2.2) lead to

$$
\begin{aligned}
& 2 \int_{0}^{l}\left[z^{T}(x, t) P_{1}+z_{t}^{T}(x, t) P_{2}\right] \triangle_{D} z(x, t) d x \\
& =2 \sum_{i=1}^{n} \int_{0}^{l}\left[P_{1}^{i} z^{i}(x, t)+P_{2}^{i} z_{t}^{i}(x, t)\right] \frac{\partial}{\partial x}\left(d_{i}(x) z_{x}^{i}(x, t)\right) d x \\
& =-2 \sum_{i=1}^{n} \int_{0}^{l} P_{1}^{i} d_{i}(x)\left(z_{x}^{i}(x, t)\right)^{2} d x-\dot{V}_{P_{2}} \\
& -\int_{0}^{l} z^{T}(x, t) P_{1} \beta z_{x}(x, t) d x=\int_{0}^{l} z_{x}^{T}(x, t) P_{1} \beta z(x, t) d x
\end{aligned}
$$

Thus, by noting that matrix $P_{1} \beta$ is diagonal, we have

$$
2 \int_{0}^{l} z^{T}(x, t) P_{1} \beta z_{x}(x, t) d x=0 .
$$

To "compensate" the cross terms in $(2.28)$ with $f_{j}(x, t-h)$ given by (2.9), for any $n \times n$ matrix $W>0$ application of Wirtinger's inequality (see e.g. (3.177) in [6]) yields

$$
\begin{aligned}
& \int_{x_{j-1}}^{x_{j}} f_{j}^{T}(x, t-h) W f_{j}(x, t-h) d x \\
& =\int_{\bar{x}_{j}}^{x_{j}}\left[\int_{\bar{x}_{j}}^{x} z_{\xi}^{T}(\xi, t-h) d \xi\right] W\left[\int_{\bar{x}_{j}}^{x} z_{\xi}(\xi, t-h) d \xi\right] d x \\
& \quad+\int_{x_{j-1}}^{\bar{x}_{j}}\left[\int_{\bar{x}_{j}}^{x} z_{\xi}^{T}(\xi, t-h) d \xi\right] W\left[\int_{\bar{x}_{j}}^{x} z_{\xi}(\xi, t-h) d \xi\right] d x \\
& \leq \frac{\Delta^{2}}{\pi^{2}} \int_{x_{j-1}}^{x_{j}} z_{x}^{T}(x, t-h) W z_{x}(x, t-h) d x, \quad j=1, \ldots, N .
\end{aligned}
$$

Thus, the following inequality holds:

$$
\begin{aligned}
& \sum_{j=1}^{N} \int_{x_{j-1}}^{x_{j}}\left[z_{x}^{T}(x, t-h) W z_{x}(x, t-h)\right. \\
& \left.\quad-\frac{\pi^{2}}{\Delta^{2}} f_{j}^{T}(x, t-h) W f_{j}(x, t-h)\right] d x \geq 0 .
\end{aligned}
$$

From (2.4) we have

$$
0 \leq \lambda_{\phi} \int_{0}^{l}\left[z^{T}(x, t) \Psi z(x, t)-\phi^{T}(z, x, t) \phi(z, x, t)\right] d x
$$

with some scalar $\lambda_{\phi}>0$.

Condition $\Upsilon_{\mathcal{N}}+\Lambda_{\mathcal{N}}+U_{\mathcal{N}} \leq 0$ in (2.15) implies $S_{2}+$ $\operatorname{He}\left\{D_{0}\left(\alpha P_{2}-P_{1}\right)\right\} \leq 0$, thus, $\alpha P_{2}-P_{1} \leq 0$. Taking into account $d_{0}^{i} \leq d_{i}(x)(i=1, \ldots, n)$ we have

$$
\begin{aligned}
& 2 \sum_{i=1}^{n} \int_{0}^{l} d_{i}(x)\left(\alpha P_{2}^{i}-P_{1}^{i}\right)\left(z_{x}^{i}(x, t)\right)^{2} d x \\
& \leq \int_{0}^{l} z_{x}^{T}(x, t) \operatorname{He}\left\{D_{0}\left(\alpha P_{2}-P_{1}\right)\right\} z_{x}(x, t) d x .
\end{aligned}
$$

Finally, in view of (2.23)-(2.27), (2.29), (2.30) and (2.33), adding the right-hand side of $(2.28)$ to $\dot{V}_{\mathcal{N}}(t)+2 \alpha V_{\mathcal{N}}(t)$ and applying S-procedure with (2.31) and (2.32), we have

$$
\begin{aligned}
& \dot{V}_{\mathcal{N}}(t)+2 \alpha V_{\mathcal{N}}(t) \\
& \leq \sum_{j=1}^{N} \int_{x_{j-1}}^{x_{j}} \eta_{\mathcal{N}, j}^{T}\left(\Upsilon_{\mathcal{N}}+\Lambda_{\mathcal{N}}\right) \eta_{\mathcal{N}, j} d x \\
& \quad-\int_{0}^{l} z_{x}^{T}(x, t-h)\left(e^{-2 \alpha h} S_{2}-W\right) z_{x}(x, t-h) d x \\
& \leq \sum_{j=1}^{N} \int_{x_{j-1}}^{x_{j}} \eta_{\mathcal{N}, j}^{T}\left(\Upsilon_{\mathcal{N}}+\Lambda_{\mathcal{N}}+U_{\mathcal{N}}\right) \eta_{\mathcal{N}, j} d x, \quad t \geq h,
\end{aligned}
$$

where we applied Wirtinger's inequality with (2.16). Here $\Upsilon_{\mathcal{N}}$ and $U_{\mathcal{N}}$ are given by $(2.17)$ and $\Lambda_{\mathcal{N}}$ is composed of (2.18). Thus, from (2.15) we have $\dot{V}_{\mathcal{N}}(t)+2 \alpha V_{\mathcal{N}}(t) \leq 0$ for all $t \geq h$ implying $V_{\mathcal{N}}(t) \leq e^{-2 \alpha(t-h)} V_{\mathcal{N}}(h)$ for all $t \geq h$. Due to the positivity of $\overline{V_{\mathcal{N}}}(t)$, there exists some $M_{1}>0$ such that

$$
V_{\mathcal{N}}(t) \geq M_{1}\|z(\cdot, t)\|_{\mathcal{H}_{0}^{1}(0, l)}^{2}, \quad t \geq h .
$$

Moreover, $V_{\mathcal{N}}(h)$ can be upper bounded by

$$
V_{\mathcal{N}}(h) \leq M_{2} \int_{0}^{h}\left[\|z(\cdot, s)\|_{\mathcal{H}_{0}^{1}(0, l)}^{2}+\left\|z_{s}(\cdot, s)\right\|_{L^{2}(0, l)}^{2}\right] d s
$$

for some $M_{2}>0$, where the bound is finite due to the wellposedness. Then (2.19) follows from (2.35) and (2.36) with $M_{0}=\frac{M_{2}}{M_{1}}$.

The feasibility of the strict inequalities (2.14)-(2.16) with $\alpha=0$ implies the feasibility of (2.14)-(2.16) with the same decision variables and with small enough $\alpha=\alpha_{0}>0$, and thus guarantees a small enough decay rate.

Remark 2.1 Note that direct substitution of $z_{t}(x, t)$ by the right-hand side of $(2.10)$ leads to the quadratic variable diffusion term $\left\|\triangle_{D} z(x, t)\right\|_{L^{2}(0, l)}^{2}$ that cannot be compensated under the Dirichlet boundary conditions (2.2). Therefore, the descriptor method (e.g. in (2.28)) allows to overcome this difficulty.

Remark 2.2 For the case of the averaged measurements $y_{j}(t)=\left\{\begin{array}{ll}0, & \text { if } t \leq h, \\ \frac{1}{\Delta_{j}} \int_{x_{j-1}}^{x_{j}} z(\xi, t-h) d \xi, & \text { if } t>h,\end{array} j=1, \ldots, N\right.$, we obtain (2.10) with

$$
f_{j}(x, t-h) \triangleq \frac{1}{\Delta_{j}} \int_{x_{j-1}}^{x_{j}}[z(x, t-h)-z(\xi, t-h)] d \xi .
$$


Since $\int_{x_{j-1}}^{x_{j}} f_{j}(\xi, t-h) d \xi=0$ and $\frac{d}{d x} f_{j}(x, t-h)=z_{x}(x, t-$ $h)$, for any $n \times n$ matrix $W>0$ the Poincaré's inequality [16] leads to

$$
\begin{aligned}
& \int_{x_{j-1}}^{x_{j}} f_{j}^{T}(x, t-h) W f_{j}(x, t-h) d x \\
& \leq \frac{\Delta^{2}}{\pi^{2}} \int_{x_{j-1}}^{x_{j}}\left[\frac{d}{d x} \sqrt{f_{j}^{T}(x, t-h) W f_{j}(x, t-h)}\right]^{2} d x \\
& \leq \frac{\Delta^{2}}{\pi^{2}} \int_{x_{j-1}}^{x_{j}}\left[\frac{d}{d x} f_{j}^{T}(x, t-h)\right] W\left[\frac{d}{d x} f_{j}(x, t-h)\right] d x, \\
& =\frac{\Delta^{2}}{\pi^{2}} \int_{x_{j-1}}^{x_{j}} z_{x}^{T}(x, t-h) W z_{x}(x, t-h) d x, \quad j=1, \ldots, N .
\end{aligned}
$$

Then, we obtain (2.31) with $f_{j}(x, t-h)$ given by $(2.37)$. Thus, LMIs of Theorem 2.1 are applicable to the case of spatially point or averaged measurements with time-delay.

Consider next $d_{i}(x) \equiv d_{0}^{i}(i=1, \ldots, n), \phi(z, x, t) \equiv 0$, $\beta=0$ and the measurement

$$
y(t)= \begin{cases}0, & \text { if } t \leq h, \\ z(x, t-h), & \text { if } t>h .\end{cases}
$$

Thus, system (2.1) becomes

$$
\begin{gathered}
z_{t}(x, t)=\triangle_{D} z(x, t)+A z(x, t)+B u(t), \\
t \geq 0, \quad x \in[0, l], \quad l>0
\end{gathered}
$$

that is stabilized by a state-feedback

$$
u(t)=-K y(t), \quad t \geq 0
$$

with $y(t)$ given by (2.38) and $K \in \mathbb{R}^{r \times n}$. Then the resulting closed-loop system has the form

$$
\begin{aligned}
z_{t}(x, t)= & D_{0} z_{x x}(x, t)+A z(x, t) \\
& -B K\left(1-\chi_{[0, h]}(t)\right) z(x, t-h), \quad t \geq 0
\end{aligned}
$$

with $D_{0}=\operatorname{diag}\left\{d_{0}^{1}, \ldots, d_{0}^{n}\right\}$ and constant matrices $A \in$ $\mathbb{R}^{n \times n}, B \in \mathbb{R}^{n \times r}, K \in \mathbb{R}^{r \times n}$ and $\chi_{[0, h]}(t)$ defined below (2.10). Since the diffusion term in (2.41) is constant, we choose $P_{1}$ in (2.28) as a non-diagonal matrix (see e.g. [23]):

$$
\begin{aligned}
& \int_{0}^{l} z^{T}(x, t) \operatorname{He}\left\{P_{1} D_{0}\right\} z_{x x}(x, t) d x \\
& =-\int_{0}^{l} z_{x}^{T}(x, t) \operatorname{He}\left\{P_{1} D_{0}\right\} z_{x}(x, t) d x .
\end{aligned}
$$

Then based on Theorem 2.1, we easily obtain the following stability result with an arbitrary $\mathcal{N} \in \mathbb{N}$ :

Corollary 2.1 Given postive scalars $h, l, \Delta$ and $\alpha$, let there exist $(\mathcal{N}+1) n \times(\mathcal{N}+1) n$ matrix $\mathcal{P}_{\mathcal{N}}, n \times n$ matrices $P_{1}, P_{2}=\operatorname{diag}\left\{P_{2}^{1}, \ldots, P_{2}^{n}\right\}>0, S_{1}>0, S_{2}>0$ and $R>0$ that satisfy (2.14) and

$$
\tilde{\Upsilon}_{\mathcal{N}}+\tilde{\Lambda}_{\mathcal{N}}+\tilde{U}_{\mathcal{N}} \leq 0
$$

where $\tilde{\Upsilon}_{\mathcal{N}}, \tilde{\Lambda}_{\mathcal{N}}=\left[\Lambda_{i j}\right]$ and $\tilde{U}_{\mathcal{N}}$ are obtained from $\Upsilon_{\mathcal{N}}, \Lambda_{\mathcal{N}}$ and $U_{\mathcal{N}}$ by setting $W=0$ and $\Lambda_{11}=\operatorname{He}\left\{P_{1} A+\frac{\pi^{2}}{l^{2}} D_{0}\left(\alpha P_{2}-\right.\right.$ $\left.\left.P_{1}\right)\right\}+S_{1}+\frac{\pi^{2}}{l^{2}} S_{2}$ and taking away the third, fifth and sixth block-columns and block-rows respectively. Then system (2.41) under the Dirichlet boundary conditions (2.2) is exponentially stable with a decay rate $\alpha>0$. Moreover, if the strict inequalities (2.14) and (2.42) are feasible with $\alpha=0$, then system (2.41) under the Dirichlet boundary conditions (2.2) is exponentially stable with a small enough decay rate $\alpha=\alpha_{0}>0$.

Remark 2.3 LMIs of Corollary 2.1 with $l=\pi$ guarantee the exponentially stability of the vector ODE with delay

$$
\dot{\bar{z}}(t)+\left(D_{0}-A\right) \bar{z}(t)+B K \bar{z}(t-h)=0 .
$$

Note that system (2.43) corresponds to the first modal dynamics (with $k=1$ ) in the modal representation of the Dirichlet boundary-value problem (2.2), (2.41) with $l=\pi$

$$
\begin{array}{r}
\dot{\bar{z}}_{k}(t)+\left(k^{2} D_{0}-A\right) \bar{z}_{k}(t)+B K \bar{z}_{k}(t-h)=0, \\
k=1,2, \ldots
\end{array}
$$

projected on the eigenfunctions of the operator $D_{0} \frac{\partial^{2}}{\partial x^{2}}$ (this operator has eigenvalues $-k^{2} D_{0}$, see e.g. [26]). The stability of (2.2), (2.41) implies the stability of (2.44). Thus, LMIs of Corollary 2.1 are tight [9].

\subsection{The dual observation problem}

Consider the semilinear diffusion equation

$$
\begin{gathered}
z_{t}(x, t)=\triangle_{D} z(x, t)-\beta z_{x}(x, t)+A z(x, t)+u(t) \\
+\sigma(z(x, t), x, t), \quad t \geq 0, \quad x \in[0, l], \quad l>0
\end{gathered}
$$

under the Dirichlet boundary conditions (2.2) with the state $z(x, t) \in \mathbb{R}^{n}$ and the control input $u(t) \in \mathbb{R}^{n}$. The diffusion term is given by (2.3) and $\sigma$ is a known function of class $\mathcal{C}^{1}$ satisfying $\sigma_{z}^{T} \sigma_{z} \leq \Psi$ with some positive definite $\Psi \in \mathbb{R}^{n \times n}$, where $\sigma_{z}$ denotes the partial derivative of a function $\sigma(z, x, t)$ with respect to $z$.

We suggest a nonlinear observer of the form

$$
\begin{aligned}
& \hat{z}_{t}(x, t)=\triangle_{D} \hat{z}(x, t)-\beta \hat{z}_{x}(x, t)+A \hat{z}(x, t)+u(t) \\
& \quad+\sigma(\hat{z}(x, t), x, t)+L \sum_{j=1}^{N} b_{j}(x)\left[y_{j}(t)-\hat{z}\left(\bar{x}_{j}, t-h\right)\right], \\
& \quad t \geq 0, \quad x \in[0, l], \quad l>0
\end{aligned}
$$

under the Dirichlet boundary conditions

$$
\hat{z}(0, t)=\hat{z}(l, t)=0,
$$

where $y_{j}(t)(j=1, \ldots, N)$ are given by $(2.7), L \in \mathbb{R}^{n \times n}$ is an observer gain and $\hat{z}(x, t)=0$ for $t \in[-h, 0]$.

Then using function $\chi_{[0, h]}(t)$ defined below $(2.10)$, the estimation error $\hat{e}(x, t)=z(x, t)-\hat{z}(x, t)$ satisfies the Dirichlet boundary value problem

$$
\begin{aligned}
\hat{e}_{t}(x, t)= & \triangle_{D} \hat{e}(x, t)-\beta \hat{e}_{x}(x, t)+A \hat{e}(x, t)+\phi(\hat{e}(x, t), x, t) \\
& -L \sum_{j=1}^{N} b_{j}(x)\left(1-\chi_{[0, h]}(t)\right) \hat{e}\left(\bar{x}_{j}, t-h\right), \quad t \geq 0,
\end{aligned}
$$


where

$$
\begin{aligned}
\phi(\hat{e}, x, t) & =\sigma(z, x, t)-\sigma(\hat{z}, x, t) \\
& =\int_{0}^{1} \sigma_{z}(\hat{z}+\xi \hat{e}, x, t) d \xi \hat{e}
\end{aligned}
$$

with

$$
\begin{aligned}
& \phi^{T}(\hat{e}, x, t) \phi(\hat{e}, x, t) \\
& \leq \hat{e}^{T}\left[\int_{0}^{1} \sigma_{z}^{T}(\hat{z}+\xi \hat{e}, x, t) \sigma_{z}(\hat{z}+\xi \hat{e}, x, t) d \xi\right] \hat{e} \\
& \leq \hat{e}^{T} \Psi \hat{e} .
\end{aligned}
$$

Note that in the latter we applied Jensen's inequality $[10,11]$. Thus, Theorem 2.1 , where $B K=L$, gives sufficient conditions for the exponential stability of (2.48) under the Dirichlet boundary conditions.

\subsection{Hierarchy of LMIs}

Following arguments for Hierarchy of LMIs in [20,21], we find that the stability conditions of Theorem 2.1 (and of Corollary 2.1 and Theorem 3.1 below) form a hierarchy of LMIs.

Theorem 2.2 Given positive scalars $h$ and $\alpha$. If LMIs of Theorem 2.1 (and of Corollary 2.1 and Theorem 3.1 below) are feasible for $\mathcal{N}$, then they are also feasible for $\mathcal{N}+1$.

Remark 2.4 Theorem 2.2 implies that LMIs of the order $\mathcal{N}+1$ lead to, at least, the same results as LMIs of the order $\mathcal{N}$. Thus, the augmented Lyapunov functional (see e.g. (2.11) and (3.7) below) with extended B-L inequality (1.5) may improve the results via the corresponding simple Lyapunov functional (i.e. (2.11) and (3.7) with $\mathcal{N}=0$ ) and Jensen's inequality [10,11].

\section{Regional stabilization of KdVB equation with an output delay}

\subsection{Problem formulation}

Consider the following KdVB equation:

$$
\begin{aligned}
& z_{t}(x, t)=-z(x, t) z_{x}(x, t)+\lambda z(x, t)+\beta z_{x x}(x, t) \\
& \quad-z_{x x x}(x, t)+\sum_{j=1}^{N} b_{j}(x) u_{j}(t), t \geq 0, x \in(0, l), l>0
\end{aligned}
$$

under the periodic boundary conditions

$$
z(0, t)=z(l, t), z_{x}(0, t)=z_{x}(l, t), z_{x x}(0, t)=z_{x x}(l, t),
$$

where $z(x, t) \in \mathbb{R}$ is the state, $u_{j}(t) \in \mathbb{R}(j=1, \ldots, N)$ are the control inputs that enter (3.1) through the shape functions $b_{j}(x)(j=1, \ldots, N)$ given by $(2.5), \lambda$ and $\beta$ are positive constants and the initial condition is given by $z(x, 0)=z_{0}(x)$. Note that for $\lambda=0$, the open-loop system (3.1) has constant solutions, whereas for $\lambda>0$ the openloop system may become unstable.
We study the KdVB equation (3.1) under the delayed stabilizing controller (2.8) with $K \in \mathbb{R}$ that leads to the closedloop system for $t \geq 0$

$$
\begin{aligned}
& z_{t}(x, t)=-z(x, t) z_{x}(x, t)+\lambda z(x, t)+\beta z_{x x}(x, t) \\
& \quad-z_{x x x}(x, t)-K\left(1-\chi_{[0, h]}(t)\right)\left[z(x, t-h)-f_{j}(x, t-h)\right],
\end{aligned}
$$

where $x \in \Omega_{j}, j=1, \ldots, N, f_{j}(x, t-h)$ is given by (2.9) and $\chi_{[0, h]}(t)$ is defined below $(2.10)$.

Now we consider the well-posedness of system (3.3). The solution of system (3.3) under the periodic boundary conditions (3.2) should be understood in the weak sense. Namely, we define the space

$$
\left\{\begin{array}{l}
\mathcal{H}_{p e r}^{1}(0, l)=\left\{g \in \mathcal{H}^{1}(0, l): g(0)=g(l)\right\}, \\
\|g\|_{\mathcal{H}_{p e r}^{1}}^{2}=\mathcal{P}_{11} \int_{0}^{l} g^{2}(x) d x+P \int_{0}^{l}\left[g^{\prime}(x)\right]^{2} d x,
\end{array}\right.
$$

where $\mathcal{P}_{11}$ and $P$ are positive constants that are related to the Lyapunov functional (see (3.7) below). Given $T>0$, a weak solution of $(3.3)$ on $[0, T]$ is a function $z(x, t) \in \mathcal{C}\left(0, T ; \mathcal{H}_{\text {per }}^{1}(0, l)\right)$ such that $z_{t} \in$ $L^{\infty}\left(0, T ; L^{2}(0, l)\right) \cap L^{2}\left(0, T ; \mathcal{H}_{p e r}^{1}(0, l)\right)$ and

$$
\begin{aligned}
& \frac{d}{d t} \int_{0}^{l} z(\xi, t) \phi(\xi) d \xi=-\int_{0}^{l} z(\xi, t) z_{x}(\xi, t) \phi(\xi) d \xi \\
& \quad+\lambda \int_{0}^{l} z(\xi, t) \phi(\xi) d \xi+\beta \int_{0}^{l} z_{\xi}(\xi, t) \phi_{\xi}(\xi) d \xi \\
& \quad-\int_{0}^{l} z_{\xi \xi}(\xi, t) \phi_{\xi}(\xi) d \xi-K \sum_{j=1}^{N} \int_{x_{j-1}}^{x_{j}}\left(1-\chi_{[0, h]}(t)\right) \\
& \quad \times[z(\xi, t-h)-f(\xi, t-h)] \phi(\xi) d \xi
\end{aligned}
$$

hold for any $\phi \in \mathcal{H}_{\text {per }}^{1}(0,1)$ and almost all $t \in[0, T]$.

The condition (3.5) is motivated via the integrationby-parts formula. Based on the Galerkin approximation method (see, e.g. [17]), one can show that (3.3) has a unique weak solution for all $T>0$ provided the initial value $z_{0} \in \mathcal{H}^{3}(0, l) \cap \mathcal{H}_{\text {per }}^{1}(0, l)$ satisfies the compatible conditions $[13]$ :

$$
z_{0}^{\prime}(0)=z_{0}^{\prime}(l), \quad z_{0}^{\prime \prime}(0)=z_{0}^{\prime \prime}(l) .
$$

\subsection{Improved regional stability conditions}

For the stability analysis of system (3.3), an augmented Lyapunov functional depending on the state $z(x, t)$ only (rather than the state derivative $z_{t}(x, t)$ ) was introduced in [13] to derive the stability conditions in terms of LMIs that allow to find an upper bound on the delay that preserves regional stability. To obtain improved regional stability conditions, we here consider a novel augmented Lyapunov functional via Legendre polynomials:

$$
\bar{V}_{\mathcal{N}}(t)=\bar{V}_{\mathcal{P}_{\mathcal{N}}}+\bar{V}_{P}+\sum_{i=1}^{2}\left(\bar{V}_{S_{i}}+\bar{V}_{R_{i}}\right), \quad \mathcal{N} \in \mathbb{N}_{0},
$$


where

$$
\begin{aligned}
& \bar{V}_{\mathcal{P}_{\mathcal{N}}}=\int_{0}^{l} \bar{\zeta}_{\mathcal{N}}^{T}(x, t) \mathcal{P}_{\mathcal{N}} \bar{\zeta}_{\mathcal{N}}(x, t) d x, \\
& \bar{V}_{P}=P \int_{0}^{l} z_{x}^{2}(x, t) d x \\
& \bar{V}_{S_{1}}=S_{1} \int_{0}^{l} \int_{t-h}^{t} e^{-2 \alpha(t-s)} z^{2}(x, s) d s d x, \\
& \bar{V}_{S_{2}}=S_{2} \int_{0}^{l} \int_{t-h}^{t} e^{-2 \alpha(t-s)} z_{x}^{2}(x, s) d s d x, \\
& \bar{V}_{R_{1}}=R_{1} \int_{0}^{l} \int_{t-h}^{t} e^{-2 \alpha(t-s)}(s-t+h) z^{2}(x, s) d s d x, \\
& \bar{V}_{R_{2}}=R_{2} \int_{0}^{l} \int_{t-h}^{t} e^{-2 \alpha(t-s)}(s-t+h) z_{x}^{2}(x, s) d s d x
\end{aligned}
$$

with

$$
\bar{\zeta}_{\mathcal{N}}(x, t)=\left[z(x, t), \Theta^{0}(x, t), \ldots, \Theta^{\mathcal{N}}(x, t)\right]^{T},
$$

and with $(\mathcal{N}+2) \times(\mathcal{N}+2)$ matrix $\mathcal{P}_{\mathcal{N}}=\left\{\mathcal{P}_{i j}\right\}$, scalars $P>$ $0, S_{i}>0, R_{i}>0(i=1,2)$ and $\alpha>0$. Here $\Theta^{k}(x, t)(k=$ $0, \ldots, \mathcal{N})$ are given by $(1.6)$. For $\mathcal{N}=0, \bar{V}_{\mathcal{N}}(t)$ coincides with the Lyapunov functional introduced in [13].

Since the solution of (3.3) does not depend on the values of $z(x, t)$ for $t<0$ [14], we redefine the initial condition to be a function

$$
z(x, t)=z_{0}(x), \quad t \leq 0 .
$$

Thus, we have

$$
\begin{aligned}
\bar{V}_{\mathcal{N}}(0)= & {\left[\mathcal{P}_{11}+2 \mathcal{P}_{12} h+\mathcal{P}_{22} h^{2}+S_{1} h+\frac{R_{1} h^{2}}{2}\right] \int_{0}^{l} z_{0}^{2}(x) d x } \\
& +\left(P+S_{2} h+\frac{R_{2} h^{2}}{2}\right) \int_{0}^{l}\left[z_{0}^{\prime}(x)\right]^{2} d x,
\end{aligned}
$$

where we applied (1.2). Note that the latter coincides with that for the case of $\mathcal{N}=0$ considered in [13]. Thus, we employ the following results borrowed from [13] that guarantee simple bounds on $\bar{V}_{\mathcal{N}}(h)$ and $z(x, t)$, and that present a solution of (3.3):

Lemma 3.1 [13] Consider system (3.3) and the functionals $\bar{V}_{\mathcal{N}}(t)$ and $\overline{\mathcal{V}}(t)$ respectively given by (3.7) and

$$
\overline{\mathcal{V}}(t)=\mathcal{P}_{11} \int_{0}^{l} z^{2}(x, t) d x+P \int_{0}^{l} z_{x}^{2}(x, t) d x
$$

with scalars $\mathcal{P}_{11}>0$ and $P>0$ that are related to the functional $\bar{V}_{\mathcal{N}}(t)$. Denote

$$
\begin{aligned}
M \triangleq \max \{ & \left(\mathcal{P}_{11}+2 \mathcal{P}_{12} h+\mathcal{P}_{22} h^{2}+S_{1} h+\frac{R_{1} h^{2}}{2}\right) \mathcal{P}_{11}^{-1}, \\
& \left.\left(P+S_{2} h+\frac{R_{2} h^{2}}{2}\right) P^{-1}\right\}+e^{2 \alpha_{1} h}-1,
\end{aligned}
$$

where $S_{i}(i=1,2)$ are from functional $\bar{V}_{\mathcal{N}}(t), h$ and $\alpha$ are positive scalars, and $\alpha_{1}$ is a positive tuning parameter. Given positive tuning parameters $C$ and $C_{1}$.

(i) Assume that along (3.3)

$$
\begin{gathered}
\dot{\overline{\mathcal{V}}}(t)-2 \alpha_{1} \overline{\mathcal{V}}(t) \leq 0, \quad t \in[0, h], \\
\dot{\bar{V}}_{\mathcal{N}}(t)+2 \alpha \bar{V}_{\mathcal{N}}(t)-2 \alpha_{1} \overline{\mathcal{V}}(t) \leq 0, \quad t \in[0, h],
\end{gathered}
$$
and

$$
M C^{2}<C_{1}^{2}
$$

hold. Then

$$
\bar{V}_{\mathcal{N}}(h) \leq M\left[\mathcal{P}_{11} \int_{0}^{l} z_{0}^{2}(x) d x+P \int_{0}^{l}\left[z_{0}^{\prime}(x)\right]^{2} d x\right]<C_{1}^{2}
$$

if $\left\|z_{0}\right\|_{\mathcal{H}_{p e r}^{1}}^{2}=\mathcal{P}_{11} \int_{0}^{l} z_{0}^{2}(x) d x+P \int_{0}^{l}\left[z_{0}^{\prime}(x)\right]^{2} d x<C^{2}$.

Assume additionally that along (3.3)

$$
\dot{\bar{V}}_{\mathcal{N}}(t)+2 \alpha \bar{V}_{\mathcal{N}}(t) \leq 0, \quad t>h
$$

holds. Then the solution of (3.3) satisfies

$$
\bar{V}_{\mathcal{N}}(t) \leq M e^{-2 \alpha(t-h)}\left[\mathcal{P}_{11} \int_{0}^{l} z_{0}^{2}(x) d x+P \int_{0}^{l}\left[z_{0}^{\prime}(x)\right]^{2} d x\right]
$$

for all $t \geq h$.

(ii) If, in addition to the conditions of (i), there exist scalars $P>0, \rho>0$ and $\varrho>0$ such that

$$
\begin{gathered}
\bar{V}_{\mathcal{N}}(t) \geq \rho \int_{0}^{l} z^{2}(x, t) d x+P \int_{0}^{l} z_{x}^{2}(x, t) d x, \\
\varrho \geq 1+\rho, \quad\left[\begin{array}{cc}
-P & 1 \\
* & -\rho
\end{array}\right]<0 .
\end{gathered}
$$

Then

$$
z(x, t) \in\left(-C_{1}, C_{1}\right) \quad \forall x \in[0,1], \quad t \geq 0 .
$$

We now present improved conditions in terms of LMIs to guarantee (3.9), (3.10), (3.12) and (3.14):

Theorem 3.1 Given positive scalars $h, l, \Delta, \alpha, K>\lambda$, and positive tuning parameters $\alpha_{1}>\lambda, C$ and $C_{1}$, let there exist scalars $(\mathcal{N}+2) \times(\mathcal{N}+2)$ matrix $\mathcal{P}_{\mathcal{N}}=\left\{\mathcal{P}_{i j}\right\}$ and scalars $P>0, S_{i}>0, R_{i}>0(i=1,2), W>0, W_{1}, \rho>0$ and $\varrho>0$ such that $(2.16)$, (3.15) and

$$
\overline{\mathcal{P}}_{\mathcal{N}}>0
$$

$$
\Xi_{\mathcal{N}}\left(-C_{1}\right) \leq 0, \quad \Xi_{\mathcal{N}}\left(C_{1}\right) \leq 0,
$$

$$
\begin{array}{ll}
\Upsilon_{\mathcal{N} 1}+\Lambda_{\mathcal{N}}\left(-C_{1}\right) \leq 0, & \Upsilon_{\mathcal{N} 1}+\Lambda_{\mathcal{N}}\left(C_{1}\right) \leq 0, \\
\Upsilon_{\mathcal{N} 2}+\Phi_{\mathcal{N}}\left(-C_{1}\right) \leq 0, & \Upsilon_{\mathcal{N} 2}+\Phi_{\mathcal{N}}\left(C_{1}\right) \leq 0,
\end{array}
$$

where

$\overline{\mathcal{P}}_{\mathcal{N}}=\mathcal{P}_{\mathcal{N}}+\operatorname{diag}\left\{-\rho, S_{1} \frac{e^{-2 \alpha h}}{h}, \ldots, S_{1} \frac{e^{-2 \alpha h}(2 \mathcal{N}+1)}{h}\right\}$,

$\Xi_{\mathcal{N}}(z)=\left[\begin{array}{ccc}-\mathcal{P}_{11}\left(\alpha_{1}-\lambda\right) & 0 & \frac{1}{2} W_{1} \\ * & -\mathcal{P}_{11} \beta-P\left(\alpha_{1}-\lambda\right)+W_{1} & \frac{1}{2} P z \\ * & * & -P \beta\end{array}\right]$

$\Upsilon_{\mathcal{N} i}=\operatorname{He}\left\{\bar{G}_{\mathcal{N} i}^{T} \mathcal{P}_{\mathcal{N}} \bar{H}_{\mathcal{N} i}\right\}+2 \alpha \bar{G}_{\mathcal{N} i}^{T} \mathcal{P}_{\mathcal{N}} \bar{G}_{\mathcal{N} i}, \quad i=1,2$,

$\bar{G}_{\mathcal{N} i}=\left[\begin{array}{cccc}1 & 0_{1 \times 3} & 0_{1 \times(\mathcal{N}+1)} & 0_{1 \times(\mathcal{N}+i)} \\ 0_{(\mathcal{N}+1) \times 1} & 0_{(\mathcal{N}+1) \times 3} & h I_{\mathcal{N}+1} & 0_{(\mathcal{N}+1) \times(\mathcal{N}+i)}\end{array}\right]$,

$\bar{H}_{\mathcal{N} i}=\left[\begin{array}{llll}0_{(2 \mathcal{N}+i+5) \times 1} & \bar{\Gamma}_{\mathcal{N} i}^{T}(0) \ldots & \bar{\Gamma}_{\mathcal{N} i}^{T}(\mathcal{N})\end{array}\right]^{T}$,

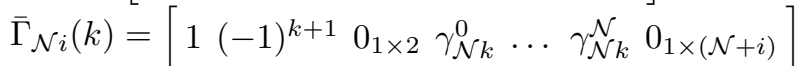


with $\gamma_{\mathcal{N} k}^{j}(j=1, \ldots, \mathcal{N})$ given by $(2.17), \Lambda_{\mathcal{N}}(z)=\left\{\Lambda_{i j}\right\}$ and $\Phi_{\mathcal{N}}(z)=\left\{\Phi_{i j}\right\}$ are symmetric matrices composed of

$$
\begin{aligned}
& \Phi_{11}=2 \mathcal{P}_{11} \lambda+S_{1}+R_{1} h, \quad \Lambda_{11}=\Phi_{11}-2 \alpha_{1} \mathcal{P}_{11}, \\
& \Phi_{12}=-\Phi_{17}=-\mathcal{P}_{11} K, \quad \Phi_{14}=\Lambda_{14}=W_{1}, \\
& \Phi_{15}=\Lambda_{15}=\left[\begin{array}{lll}
\mathcal{P}_{12} & \ldots & \mathcal{P}_{1(\mathcal{N}+2)}
\end{array}\right] h \lambda, \\
& \Phi_{22}=\Lambda_{22}=-S_{1} e^{-2 \alpha h}, \quad \Phi_{24}=-\Phi_{47}=P K, \\
& \Phi_{25}=-\Phi_{57}^{T}=-\left[\begin{array}{lll}
\mathcal{P}_{12} & \ldots & \mathcal{P}_{1(\mathcal{N}+2)}
\end{array}\right] h K, \\
& \Phi_{33}=-2 \mathcal{P}_{11} \beta+2 P \lambda+2 \alpha P+S_{2}+R_{2} h+2 W_{1}, \\
& \Lambda_{33}=\Phi_{33}-2 \alpha_{1} P, \quad \Phi_{34}=\Lambda_{34}=P z, \\
& \Phi_{35}=\Lambda_{35}=-\left[\begin{array}{lll}
\mathcal{P}_{12} & \ldots & \mathcal{P}_{1(\mathcal{N}+2)}
\end{array}\right] h z, \\
& \Phi_{36}=\Lambda_{36}=-\left[\begin{array}{lll}
\mathcal{P}_{12} & \ldots & \mathcal{P}_{1(\mathcal{N}+2)}
\end{array}\right] h \beta, \\
& \Phi_{44}=\Lambda_{44}=-2 P \beta, \quad \Phi_{77}=-W \frac{\pi^{2}}{\Delta^{2}}, \\
& \Phi_{46}=\Lambda_{46}=\left[\begin{array}{lll}
\mathcal{P}_{12} & \ldots & \mathcal{P}_{1(\mathcal{N}+2)}
\end{array}\right] h, \\
& \Phi_{55}=\Lambda_{55}=-R_{1} h e^{-2 \alpha h} \operatorname{diag}\{1, \ldots, 2 \mathcal{N}+1\}, \\
& \Phi_{66}=\Lambda_{66}=-R_{2} h e^{-2 \alpha h} \operatorname{diag}\{1, \ldots, 2 \mathcal{N}+1\},
\end{aligned}
$$

other blocks are zero matrices. If (3.11) holds, then for any initial state $z_{0} \in \mathcal{H}^{3}(0, l) \cap \mathcal{H}_{\text {per }}^{1}(0, l)$ satisfying the compatible conditions (3.6) with $\left\|z_{0}\right\|_{\mathcal{H}_{\text {per }}^{1}}<C$, system (3.3) under the periodic boundary conditions (3.2) possesses a unique weak solution in the sense that for any $T>0$, $z(x, t) \in \mathcal{C}\left(0, T ; \mathcal{H}_{\text {per }}^{1}(0, l)\right)$. Moreover, the solution of $(3.3)$ satisfies (3.13) for all $t \geq h$.

Proof: First, Lemma 1.1 ensures the following inequality

$$
\bar{V}_{\mathcal{P}_{\mathcal{N}}}+\bar{V}_{S_{1}} \geq \int_{0}^{l} \bar{\zeta}_{\mathcal{N}}^{T}(x, t) \overline{\mathcal{P}}_{\mathcal{N}} \bar{\zeta}_{\mathcal{N}}(x, t)+\rho \int_{0}^{l} z^{2}(x, t) d x,
$$

where matrix $\overline{\mathcal{P}}_{\mathcal{N}}>0$ is given in (3.21) and guarantees

$$
\begin{aligned}
\bar{V}_{\mathcal{N}}(t) & \geq \bar{V}_{\mathcal{P}_{\mathcal{N}}}+\bar{V}_{S_{1}}+P \int_{0}^{l} z_{x}^{2}(x, t) d x \\
& \geq \rho \int_{0}^{l} z^{2}(x, t) d x+P \int_{0}^{l} z_{x}^{2}(x, t) d x,
\end{aligned}
$$

which, together with $\rho>0$ and $P>0$, implies the positivity of $\bar{V}_{\mathcal{N}}(t)$. In the next step, the objective is to show that LMIs (3.18)-(3.20) guarantee (3.9), (3.10) and (3.12) respectively.

For any $t \geq 0$, differentiating $\bar{V}_{\mathcal{P}_{\mathcal{N}}}$ along the trajectories of (3.3) yields

$$
\begin{aligned}
\dot{\bar{V}}_{\mathcal{P}_{\mathcal{N}}}= & 2 \int_{0}^{l} \bar{\zeta}_{\mathcal{N}}^{T}(x, t) \mathcal{P}_{\mathcal{N}} \dot{\bar{\zeta}}_{\mathcal{N}}(x, t) d x \\
= & \int_{0}^{l} \bar{\eta}_{\mathcal{N}}^{T} \bar{\Upsilon}_{\mathcal{N} 1} \bar{\eta}_{\mathcal{N}} d x+2 \mathcal{P}_{11} \int_{0}^{l} z(x, t) z_{t}(x, t) d x \\
& +2 \sum_{k=2}^{\mathcal{N}+2} \mathcal{P}_{1 k} \int_{0}^{l} \Theta^{k-2}(x, t) z_{t}(x, t) d x
\end{aligned}
$$

where (3.5) has been used with $\phi=z$, and with $\bar{\Upsilon}_{\mathcal{N} 1}$ given by (3.3) and the augmented vector

$$
\begin{aligned}
\bar{\eta}_{\mathcal{N}}= & {\left[z(x, t), z(x, t-h), z_{x}(x, t), z_{x x}(x, t), \frac{1}{h} \Theta^{0}(x, t),\right.} \\
& \left.\ldots, \frac{1}{h} \Theta^{\mathcal{N}}(x, t), \frac{1}{h} \Theta_{x}^{0}(x, t), \ldots, \frac{1}{h} \Theta_{x}^{\mathcal{N}}(x, t)\right]^{T} .
\end{aligned}
$$

Substituting $z_{t}(x, t)$ by the right-hand side of (3.3) in (3.23), integrating by parts and using the periodic boundary conditions (3.2) lead to

$$
\begin{aligned}
& \dot{\bar{V}}_{\mathcal{P}_{\mathcal{N}}}=\int_{0}^{l} \bar{\eta}_{\mathcal{N}}^{T}(x, t) \bar{\Upsilon}_{\bar{\eta}}(x, t) d x \\
& \quad+2 \mathcal{P}_{11} \lambda \int_{0}^{l} z^{2}(x, t) d x-2 \mathcal{P}_{11} \beta \int_{0}^{l} z_{x}^{2}(x, t) d x \\
& \quad+2 \sum_{k=2}^{\mathcal{N}+2} \mathcal{P}_{1 k} \lambda \int_{0}^{l} z(x, t) \Theta^{k-2}(x, t) d x \\
& \quad-2 \sum_{k=2}^{\mathcal{N}+2} \mathcal{P}_{1 k} \int_{0}^{l} z(x, t) z_{x}(x, t) \Theta^{k-2}(x, t) d x \\
& \quad-2 \sum_{k=2}^{\mathcal{N}+2} \mathcal{P}_{1 k} \beta \int_{0}^{l} z_{x}(x, t) \Theta_{x}^{k-2}(x, t) d x \\
& \quad+2 \sum_{k=2}^{\mathcal{N}+2} \mathcal{P}_{1 k} \int_{0}^{l} z_{x x}(x, t) \Theta_{x}^{k-2}(x, t) d x \\
& \quad-2 K\left(1-\chi_{[0, h]}(t)\right) \sum_{j=1}^{N} \int_{x_{j-1}}^{x_{j}}\left[\mathcal{P}_{11} z(x, t)\right. \\
& \left.\quad+\sum_{k=2}^{\mathcal{N}+2} \mathcal{P}_{1 k} \Theta^{k-2}(x, t)\right]\left[z(x, t-h)-f_{j}(x, t-h)\right] d x .
\end{aligned}
$$

Concerning the other terms of the Lyapunov functional, we have

$$
\begin{aligned}
\dot{\bar{V}}_{P}= & 2 P \int_{0}^{l} z_{x}(x, t) z_{x t}(x, t) d x \\
= & -2 P \int_{0}^{l} z_{x x}(x, t) z_{t}(x, t) d x \\
= & 2 P \lambda \int_{0}^{l} z_{x}^{2}(x, t) d x-2 P \beta \int_{0}^{l} z_{x x}^{2}(x, t) d x \\
& +2 P \int_{0}^{l} z(x, t) z_{x}(x, t) z_{x x}(x, t) d x \\
& +2 P K\left(1-\chi_{[0, h]}(t)\right) \sum_{j=1}^{N} \int_{x_{j}-1}^{x_{j}} z_{x x}(x, t) \\
& \times\left[z(x, t-h)-f_{j}(x, t-h)\right] d x
\end{aligned}
$$

and

$$
\begin{gathered}
\dot{\bar{V}}_{S_{1}}+2 \alpha \bar{V}_{S_{1}}=S_{1} \int_{0}^{l} z^{2}(x, t) d x \\
\quad-S_{1} e^{-2 \alpha h} \int_{0}^{l} z^{2}(x, t-h) d x, \\
\dot{\bar{V}}_{S_{2}}+2 \alpha \bar{V}_{S_{2}}=S_{2} \int_{0}^{l} z_{x}^{2}(x, t) d x \\
\quad-S_{2} e^{-2 \alpha h} \int_{0}^{l} z_{x}^{2}(x, t-h) d x .
\end{gathered}
$$

Then the application of Lemma 1.1 leads to

$$
\begin{aligned}
& \dot{\bar{V}}_{R_{1}}+2 \alpha \bar{V}_{R_{1}}=R_{1} h \int_{0}^{l} z^{2}(x, t) d x \\
& \quad-R_{1} \int_{0}^{l} \int_{t-h}^{t} e^{-2 \alpha(t-s)} z^{2}(x, s) d s d x \\
& \leq R_{1} h \int_{0}^{l} z^{2}(x, t) d x \\
& \quad-R_{1} e^{-2 \alpha h} \sum_{k=0}^{\mathcal{N}} \frac{2 k+1}{h} \int_{0}^{l}\left[\Theta^{k}(x, t)\right]^{2} d x,
\end{aligned}
$$

and

$$
\begin{aligned}
& \dot{\bar{V}}_{R_{2}}+2 \alpha \bar{V}_{R_{2}}=R_{2} h \int_{0}^{l} z_{x}^{2}(x, t) d x \\
& \quad-R_{2} \int_{0}^{l} \int_{t-h}^{t} e^{-2 \alpha(t-s)} z_{x}^{2}(x, s) d s d x \\
& \leq R_{2} h \int_{0}^{l} z_{x}^{2}(x, t) d x \\
& \quad-R_{2} e^{-2 \alpha h} \sum_{k=0}^{\mathcal{N}} \frac{2 k+1}{h} \int_{0}^{l}\left[\Theta_{x}^{k}(x, t)\right]^{2} d x .
\end{aligned}
$$

Additionally, we note that for any $W_{1}$ in $\mathbb{R}$, we have

$$
2 W_{1} \int_{0}^{l}\left[z(x, t) z_{x x}(x, t)+z_{x}^{2}(x, t)\right] d x=0 .
$$

Two cases may occur. When $t$ is in the first delay interval $[0, h]$ (i.e. $\left.\chi_{[0, h]}(t)=1\right)$, then adding $(3.31)$ to $\dot{\bar{V}}_{\mathcal{N}}(t)+$ $2 \alpha \bar{V}_{\mathcal{N}}(t)$ yields, in light of (3.23)-(3.30), for all $t \in[0, h]$ $\dot{\bar{V}}_{\mathcal{N}}(t)+2 \alpha \bar{V}_{\mathcal{N}}(t)-2 \alpha_{1} \mathcal{V}(t) \leq \int_{0}^{l} \bar{\eta}_{\mathcal{N}}^{T}\left[\Upsilon_{\mathcal{N} 1}+\Lambda_{\mathcal{N}}(z)\right] \bar{\eta}_{\mathcal{N}} d x$ 
When $t>h$ (i.e. $\chi_{[0, h]}(t)=0$ ), we additionally apply an S-procedure with $(2.31)$, with parameter $W>0$, to compensate for $f_{j}(x, t-h)$ in $(2.9)$ that leads to

$$
\begin{aligned}
& \dot{\bar{V}}_{\mathcal{N}}(t)+2 \alpha \bar{V}_{\mathcal{N}}(t) \\
& \leq \sum_{j=1}^{N} \int_{x_{j-1}}^{x_{j}} \tilde{\eta}_{\mathcal{N}, j}^{T}\left[\Upsilon_{\mathcal{N} 2}+\Phi_{\mathcal{N}}(z)\right] \tilde{\eta}_{\mathcal{N}, j} d x \\
& \quad-\left(S_{2} e^{-2 \alpha h}-W\right) \int_{0}^{l} z_{x}^{2}(x, t-h) d x \\
& \leq \sum_{j=1}^{N} \int_{x_{j-1}}^{x_{j}} \tilde{\eta}_{\mathcal{N}, j}^{T}\left[\Upsilon_{\mathcal{N} 2}+\Phi_{\mathcal{N}}(z)\right] \tilde{\eta}_{\mathcal{N}, j} d x, \quad t>h,
\end{aligned}
$$

where we applied (2.16). Here $\Upsilon_{\mathcal{N} i}(i=1,2)$ and $\bar{\eta}_{\mathcal{N}}$ are respectively given by $(3.21)$ and $(3.24), \tilde{\eta}_{\mathcal{N}, j}=\left[\bar{\eta}_{\mathcal{N}}^{T}, f_{j}(x, t-\right.$ $h)]^{T}$, and $\Lambda_{\mathcal{N}}(z)$ and $\Phi_{\mathcal{N}}(z)$ are symmetric matrices composed of (3.22).

Similarly, differentiating $\overline{\mathcal{V}}(t)$ in (3.8) along the trajectories of (3.3) yields

$$
\dot{\overline{\mathcal{V}}}(t)-2 \alpha_{1} \overline{\mathcal{V}}(t)=2 \int_{0}^{l} \kappa^{T} \Xi_{\mathcal{N}}(z) \kappa d x, \quad t \in[0, h],
$$

where $\kappa=\left[z(x, t), z_{x}(x, t), z_{x x}(x, t)\right]^{T}$ and $\Xi_{\mathcal{N}}(z)$ is given by $(3.21)$.

Since $\Xi_{\mathcal{N}}(z), \Lambda_{\mathcal{N}}(z)$ and $\Phi_{\mathcal{N}}(z)$ are affine in $z, \Xi_{\mathcal{N}}(z) \leq 0$, $\Upsilon_{\mathcal{N} 1}+\Lambda_{\mathcal{N}}(z) \leq 0$ and $\Upsilon_{\mathcal{N} 2}+\Phi_{\mathcal{N}}(z) \leq 0$ hold for all $z \in\left(-C_{1}, C_{1}\right)$ given by (3.16) if LMIs (3.18)-(3.20) are feasible. From (3.32)-(3.34), it follows that LMIs (3.18)(3.20) guarantee $(3.9),(3.10)$ and (3.12) respectively.

Finally, assertion of Theorem 3.1 follows from Lemma 3.1.

\section{Numerical examples}

Example 4.1 Consider the chemical reactor model from [18,22] governed by heat equation (2.1) under the Dirichlet boundary conditions (2.2) with the measurements (2.7), where $n=2, r=1, l=10, D_{0}=\operatorname{diag}\{0.01,0.005\}$, $\beta=\operatorname{diag}\{0.011,1.1\}, \phi=\left[\phi_{1}\left(z^{1}\right), 0\right]^{T}, \Psi=\operatorname{diag}\left\{10^{-4}, 0\right\}$ and

$$
A=\left[\begin{array}{cc}
0 & 0.01 \\
-0.45 & -0.2
\end{array}\right], \quad B=\left[\begin{array}{l}
1 \\
1
\end{array}\right], \quad K=\left[\begin{array}{ll}
1 & 0
\end{array}\right] .
$$

This model accounts for an activator temperature $z^{1}$ that undergoes reaction, advection and diffusion, and for a fast inhibitor concentration $z^{2}$, which may be advected by the flow [18].

Assume that there are $N=20$ in-domain sensors transmitting measurements (2.7) at $\bar{x}_{j}=\frac{2 j-1}{2 N}, j=1, \ldots, N$ (the centers of $\left.\Omega_{j}=\left[\frac{j-1}{N}, \frac{j}{N}\right)\right)$ implying $\Delta=0.5$. LMIs of Theorem 2.1 with various values of $\mathcal{N}$ and $\alpha=0$ lead to the maximal value of $h$ (see Table 4.1) that preserves the stability. As expected, better results are obtained as the degree of the polynomial $\mathcal{N}$ increases, but at the cost of additional decision variables. Moreover, the maximal value of $h$ remains
Table 4.1

Maximal value of $h$ via Theorem 2.1 (Example 4.1).

\begin{tabular}{lll}
\hline & $h_{\max }$ & No. of decision variables \\
\hline $\mathcal{N}=0$ & 0.5139 & $\frac{5 n^{2}+9 n+2}{2}$ \\
$\mathcal{N}=1$ & 0.5904 & $4 n^{2}+5 n+1$ \\
$\mathcal{N} \geq 2$ & 0.5909 & $\frac{\left(\mathcal{N}^{2}+2 \mathcal{N}+5\right) n^{2}+(\mathcal{N}+9) n+2}{2}$ \\
\hline
\end{tabular}

as 0.5909 when $\mathcal{N} \geq 2$. Clearly, an improvement close to $15 \%$ is achieved.

Example 4.2 Consider heat equation (2.41) under the Dirichlet boundary conditions (2.2) with $l=\pi, D_{0}=I_{2}$ and

$$
A=\left[\begin{array}{cc}
1 & 1 \\
-1 & 0
\end{array}\right], \quad B K=\left[\begin{array}{ll}
0 & 0 \\
0 & 1
\end{array}\right]
$$

LMIs of Corollary 2.1 with various values of $\mathcal{N}$ and $\alpha=$ 0 lead to the maximal value of $h$ shown in Table 4.2. As explained in Remark 2.3, the latter results correspond also to the stability of the following ODE with time-delay

$$
\dot{\bar{z}}(t)+\left[\begin{array}{rr}
0 & -1 \\
1 & 1
\end{array}\right] \bar{z}(t)+\left[\begin{array}{ll}
0 & 0 \\
0 & 1
\end{array}\right] \bar{z}(t-h)=0 .
$$

Note that the analytical upper bound for the asymptotic stability of the latter ODE is $h=\pi$ (see Chapter 2.3.2 in [6]). It is clear that our method recovers the analytical upper bound with a finite degree $\mathcal{N}=4$ of the polynomials.

Example 4.3 Consider KdVB equation (3.1) under the periodic boundary conditions (3.2) with $\lambda=15, \beta=0.5$ and $l=1$. Assume that there are $N=10$ in-domain sensors transmitting point measurements $(2.7)$ at $\bar{x}_{j}=\frac{2 j-1}{2 N}$, $j=1, \ldots, N$ implying $\Delta=0.1$. By using the point measurements (2.7), we study system (3.1) under the control law (2.8) with $K=20$.

LMIs of Theorem 3.1 with various values of $\mathcal{N}, \alpha=1, \alpha_{1}=$ $20, C=0.044$ and $C_{1}=0.05$ lead to the maximal value of $h$ (see Table 4.3) that preserves the exponential stability of the closed-loop system for any initial values satisfying $\left\|z_{0}\right\|_{\mathcal{H}_{\text {per }}^{1}}<0.044$. It is clear that the improvement on the upper bound of delay is achieved as the degree $\mathcal{N}$ of the polynomial increases and the maximal value of $h$ remains as $2.4442 \times 10^{-3}$ when $\mathcal{N} \geq 3$. We find also the feasible solutions of the LMIs, e.g.

$$
\mathcal{N}=3: \quad \mathcal{P}_{11}=189.4785, \quad P=2.1978 .
$$

The latter allows to choose a larger initial condition (comparatively to $z_{0}(x)=0.0025 \sin (2 \pi x), 0 \leq x \leq 1$ in [13]), e.g. $z_{0}(x)=0.0037 \sin (2 \pi x), 0 \leq x \leq 1$ satisfying

$$
\begin{aligned}
\left\|z_{0}\right\|_{\mathcal{H}_{p e r}^{1}}^{2} & =189.4785\left\|z_{0}\right\|_{L^{2}(0,1)}^{2}+2.1978\left\|z_{0}^{\prime}\right\|_{L^{2}(0,1)}^{2} \\
& <0.044^{2}
\end{aligned}
$$


Table 4.2

Maximal value of $h$ via Corollary 2.1 (Example 4.2).

\begin{tabular}{llllll}
\hline & $\mathcal{N}=0$ & $\mathcal{N}=1$ & $\mathcal{N}=2$ & $\mathcal{N}=3$ & $\mathcal{N} \geq 4$ \\
\hline$h_{\max }$ & 1.9999 & 3.0344 & 3.1362 & 3.1414 & 3.1415 \\
\hline
\end{tabular}

Table 4.3

Maximal value of $h$ via Theorem (3.1) (Example 4.3).

\begin{tabular}{rl}
\hline & $h_{\max }$ \\
\hline Kang \& Fridman $[13]$ & $1.9 \times 10^{-3}$ \\
\hline Theorem 3.1 with $\mathcal{N}=0$ & $1.9 \times 10^{-3}$ \\
$\mathcal{N}=1$ & $2.4431 \times 10^{-3}$ \\
$\mathcal{N}=2$ & $2.4437 \times 10^{-3}$ \\
$\mathcal{N} \geq 3$ & $2.4442 \times 10^{-3}$ \\
\hline
\end{tabular}

\section{Conclusions}

In this paper, stabilization of heat equation and KdVB equation under constant output delay has been studied. By constructing two augmented Lyapunov functionals that respectively depend on the state derivative and the state only, sufficient conditions in terms of LMIs that preserve the exponential stability have been derived. The resulting LMI conditions show improvements in numerical examples. The suggested augmented Lyapunov functionals can be used for delayed control of various PDEs via spatial decomposition method: Kuramoto-Sivashinskii equation (as considered in [12]), damped wave and beam equations (see e.g. [24,25]).

\section{References}

[1] T. Ahmed-Ali, E. Fridman, F. Giri, M. Kahelras, F. LamnabhiLagarrigue, and L. Burlion. Observer design for a class of parabolic systems with large delays and sampled measurements. IEEE Transactions on Automatic Control, 65(5):2200-2206, 2020.

[2] N. Bar Am and E. Fridman. Network-based $H_{\infty}$ filtering of parabolic systems. Automatica, 50(12):3139-3146, 2014.

[3] L. Baudouin, A. Seuret, and F. Gouaisbaut. Stability analysis of a system coupled to a heat equation. Automatica, 99:195$202,2019$.

[4] R. F. Curtain and H. Zwart. An introduction to infinitedimensional linear systems theory. Springer, New York, 1995.

[5] H. Demiray. A travelling wave solution to the KdV-Burgers equation. Applied mathematics and computation, 154(3):665670, 2004.

[6] E. Fridman. Introduction to time-delay systems: analysis and control. Birkhäuser, Basel, 2014.

[7] E. Fridman and N. Bar Am. Sampled-data distributed $H_{\infty}$ control of transport reaction systems. SIAM Journal on Control and Optimization, 51(2):1500-1527, 2013.

[8] E. Fridman and A. Blighovsky. Robust sampled-data control of a class of semilinear parabolic systems. Automatica, 48:826836, 2012.

[9] E. Fridman and Y. Orlov. Exponential stability of linear distributed parameter systems with time-varying delays. Automatica, 45:194-201, 2009.
[10] K. Gu. An integral inequality in the stability problem of timedelay systems. In Proceedings of the 39th IEEE Conference on Decision and Control, pages 2805-2810. IEEE, 2000.

[11] K. Gu, J. Chen, and V. L. Kharitonov. Stability of time delay systems. Birkhäuser, Boston, 2003.

[12] W. Kang and E. Fridman. Distributed sampled-data control of Kuramoto-Sivashinsky equation. Automatica, 95:514-524, 2018.

[13] W. Kang and E. Fridman. Distributed stabilization of Korteweg-de Vries-Burgers equation in the presence of input delay. Automatica, 100:260-273, 2019.

[14] K. Liu and E. Fridman. Delay-dependent methods and the first delay interval. Systems \& Control Letters, 64:57-63, 2014.

[15] P. Park, W. I. Lee, and S. Y. Lee. Auxiliary functionbased integral inequalities for quadratic functions and their applications to time-delay systems. Journal of the Franklin Institute, 352(4):1378-1396, 2015.

[16] L. E. Payne and H. F. Weinberger. An optimal poincaré inequality for convex domains. Archive for Rational Mechanics and Analysis, 5(1):286-292, 1960.

[17] A. Pisano and Y. Orlov. On the ISS properties of a class of parabolic DPS' with discontinuous control using sampled-inspace sensing and actuation. Automatica, 81:447-454, 2017.

[18] A. Selivanov and E. Fridman. Distributed event-triggered control of diffusion semilinear PDEs. Automatica, 68:344-351, 2016.

[19] A. Seuret and F. Gouaisbaut. Wirtinger-based integral inequality: application to time-delay systems. Automatica, 49(9):2860-2866, 2013.

[20] A. Seuret and F. Gouaisbaut. Hierarchy of LMI conditions for the stability analysis of time-delay systems. Systems 83 Control Letters, 81:1-7, 2015.

[21] A. Seuret, F. Gouaisbaut, and Y. Ariba. Complete quadratic Lyapunov functionals for distributed delay systems. Automatica, 62:168-176, 2015.

[22] Y. Smagina and M. Sheintuch. Using Lyapunov's direct method for wave suppression in reactive systems. Systems \& Control Letters, 55(7):566-572, 2006.

[23] O. Solomon and E. Fridman. Stability and passivity analysis of semilinear diffusion PDEs with time-delays. International Journal of Control, 88(1):180-192, 2015.

[24] M. Terushkin and E. Fridman. Network-based control of a semilinear damped beam equation under point and pointlike measurements. Systems \& Control Letters, 136:104617, 2020.

[25] M. Terushkin and E. Fridman. Network-based deployment of nonlinear multi agents over open curves: a PDE approach. Automatica, 129:109697, 2021.

[26] J. Wu. Theory and applications of partial functional differential equations. f Springer-Verlag, New York, 1996.

[27] H.-B. Zeng, Y. He, M. Wu, and J. She. Free-matrix-based integral inequality for stability analysis of systems with timevarying delay. IEEE Transactions on Automatic Control, 60(10):2768-2772, 2015 .

[28] J. Zhang, W. Kang, E. Fridman, and A. Seuret. Improved observer design for heat equation with constant measurement delay via Legendre polynomials. In Proceedings of the 59th IEEE Conference on Decision and Control, pages 4448-4453, 2020. 